\title{
Independent risk factors and feasibility of ultrasound diagnosis of ultrasound-guided non-cytologically diagnostic thyroid nodules
}

\author{
Cheng Jiang, Jiandong Chang, Xiaoyan Chen, Hao Zhang, Meina Xu \\ Department of Ultrasound, Xiamen Hospital of Traditional Chinese Medicine, Xiamen, China \\ Contributions: (I) Conception and design: C Jiang, J Chang; (II) Administrative support: J Chang; (III) Provision of study materials or patients: C \\ Jiang, X Chen; (IV) Collection and assembly of data: X Chen, H Zhang; (V) Data analysis and interpretation: C Jiang, X Chen; (VI) Manuscript \\ writing: All authors; (VII) Final approval of manuscript: All authors. \\ Correspondence to: Jiandong Chang, MD. Department of Ultrasound, Xiamen Hospital of Traditional Chinese Medicine, 1739 Xianyue Road, Xiamen \\ 361000, China. Email: CJD20005@126.com.
}

Background: To explore the independent risk factors and feasibility of ultrasound diagnosis of ultrasoundguided non-cytologically diagnostic thyroid nodules.

Methods: This study included 200 patients with thyroid nodules that were diagnosed or suspected of being Thyroid Imaging Reporting and Data System (TI-RADS) 4 nodules between January 2017 and January 2019. All patients received surgical treatment and pathological diagnosis, and were divided into a cytologically diagnostic group and a non-cytologically diagnostic group based on whether they could be diagnosed by ultrasound-guided fine needle aspiration cytology (UG-FNAC). Patients were further divided into benign and malignant groups according to the results of surgical pathology. Logistic regression analysis was used to clarify the risk factors that could not be cytologically diagnosed in TI-RADS 4 nodules. For the diagnosis of benign and malignant nodules, we combined contrast-enhanced ultrasound (CEUS) and ultrasound elastography (UE) to establish a joint scoring strategy. The diagnostic value of the joint scoring strategy was evaluated by receiver operating characteristic (ROC) curve.

Results: A total of 216 TI-RADS type 4 nodules were detected in 200 patients. Among them, 40 nodules were included in the non-cytologically diagnostic group, and 176 nodules were included in the cytologically diagnostic group. The multi-parameter logistic regression showed that: aspect ratio <1, irregular edge, scattered coarse calcification, middle layer, and lower layer were independent influencing factors leading to undiagnosed puncture cytology. Among the 216 nodules in 200 patients, 168 were pathologically diagnosed as malignant nodules (malignant group), and 48 nodules were diagnosed as benign nodules (benign group). According to the joint scoring strategy, the distribution of comprehensive scores changes significantly at 5-6 scores while in malignant nodules, the distribution of comprehensive scores changes significantly at 6-7 scores. ROC curve analysis showed that the diagnostic value was the highest when ' 6 scores' was used as the critical point for diagnosis [area under curve $(\mathrm{AUC})=0.893, \mathrm{P}<0.05]$.

Conclusions: The combined scoring strategy of CEUS and UE was an effective method to diagnose TIRADS4 nodules could not be diagnosed by UG-FNAC.

Keywords: Contrast-enhanced ultrasound (CEUS); ultrasound elastography (UE); thyroid nodules; Thyroid Imaging Reporting and Data System (TI-RADS); ultrasound-guided fine needle aspiration cytology (UG-FNAC)

Submitted Jul 02, 2021. Accepted for publication Sep 17, 2021.

doi: $10.21037 /$ gs-21-512

View this article at: https://dx.doi.org/10.21037/gs-21-512 


\section{Introduction}

Thyroid diseases have exhibited a significant upward trend in recent years $(1,2)$. Thyroid nodules could be found in $70 \%$ of the population by ultrasound examination of the neck (3). The malignant risk of Thyroid Imaging Reporting and Data System (TI-RADS) 4 nodules was confirmed to be $5-80 \%$ (4), as it was difficult to accurately define benign and malignant lesions. Patients with TIRADS 4 thyroid nodules were previously recommended for qualitative puncture or surgical treatment (5). Therefore, the differentiation of benign and malignant nodules is key to the formulation of clinical diagnosis and treatment strategies; accurate diagnosis may reduce some unnecessary surgical treatments.

Ultrasound-guided fine needle aspiration cytology (UG-FNAC) was considered to be the most valuable method for preoperative diagnosis of benign and malignant thyroid nodules (6). However, due to various factors, the undiagnosed rate was approximately $10-20 \%(7,8)$, which affected further clinical diagnosis and treatment. It is necessary to further diagnose TI-RADS 4 thyroid nodules which failed in UG-FNAC diagnosis. Obviously, UGFNAC is an invasive diagnostic method. The advantage of ultrasound is that it is non-invasive and the cost of the inspection is lower. If we can find an effective way to realize the diagnosis of TI-RADS 4 thyroid nodules, which cannot be diagnosed by UG-FNAC directly through ultrasound, it will be of great significance. Contrast-enhanced ultrasound (CEUS) performs qualitative diagnosis of nodules indirectly by dynamically observing the distribution of microvessels in the target and the characteristics of blood perfusion in realtime. Ultrasound elastography (UE) reflects the softness and hardness information of the lesion through the degree of deformation. The greater the hardness of the nodule, the more malignant it will be. Although CEUS and UE have high value in the qualitative diagnosis of nodules, the accuracy of using only one of them for the clinical diagnosis of TI-RADS 4 types of nodules is still not ideal $(8,9)$. To achieve the purpose of improving diagnosis, the diagnosis of TI-RADS 4 nodules was based on direct observations of the lesion signals in the previous study about the combined diagnosis of TI-RADS 4 nodules by CEUS and UE (10-12), which obviously produced diagnostic bias.

In this study, we clarified the independent risk factors of non-cytologically diagnostic TI-RADS 4 thyroid nodules, including aspect ratio $<1$, irregular edge, scattered coarse calcification, middle layer, and lower layer. And we established a joint scoring strategy combining CEUS and UE for the diagnosis of these nodules. Our research confirms that CEUS combined with UE can effectively diagnose TI-RADS category $4 \mathrm{a}$ and $4 \mathrm{~b}$ nodules that UGFNAC cannot diagnose, which has important clinical value. We present the following article in accordance with the STARD reporting checklist (available at https://dx.doi. org/10.21037/gs-21-512).

\section{Methods}

\section{Patients}

200 patients with thyroid nodules that were diagnosed or suspected of being TI-RADS type 4 nodules between January 2017 and January 2019 were admitted. The inclusion criteria were as follows: (I) according to the TIRADS standard, the five items of solid nodules, including hypoechoic or very low echo, irregular edges, aspect ratio $>1$, and microcalcification, were regarded as the sonographic features of malignant nodules, and those satisfying $1-4$ were classified in the TI-RADS4 category (13); (II) according to the Bethesda cytology classification, the results of the first UG-FNAC were selected for evaluation (14); (III) patients who underwent CEUS and UE within 3-12 months of follow-up after the first UG-FNAC diagnosis of the nodules was unclear; and (IV) benign and malignant judgment of nodules was clearly based on surgical pathology. The exclusion criteria were as follows: (I) patients underwent UG-FNAC again but no surgical pathological results; (II) patients with a history of partial thyroidectomy or severe diffuse disease; and (III) patients with missing clinical data. All procedures performed in this study involving human participants were in accordance with the Declaration of Helsinki (as revised in 2013). This study was approved by the Medical Ethics Committee of Xiamen hospital of traditional Chinese medicine (No. EC20200808A) and informed consent was taken from all the patients.

\section{$U G-F N A C$}

Patients underwent routine blood and coagulation function tests before surgery. Patients lay in the supine position and the drapes were routinely disinfected. The patients were then instructed to avoid swallowing, $2 \%$ lidocaine was used for local anesthesia, and a $23 \mathrm{G}$ puncture needle was used to penetrate into the thyroid nodule, clearly illustrating that the needle tip was in the lesion area. The needle was lifted 
back and forth 5-7 times in different needle paths, and was subsequently pulled out. Two experienced pathologists stained and evaluated the cells in real time. The number of punctures was usually 1-3 stitches. According to the standard of the Bethesda system for reporting thyroid cytopathology, the thyroid nodule was divided into categories I-VI: undiagnosable or insufficient cellular components, benign, ambiguous or atypical follicular lesions, follicular tumors, suspiciously malignant, and malignant.

\section{CEUS and UE}

\section{Equipment}

The MyLab Twice color Doppler ultrasonic diagnostic instrument (Esaote, Italy) was used; probe model: LA523, frequency: 5-13 MHz, with real-time elastography function. Ultrasound contrast used the following: probe model LA522; frequency: is 3-9 MHz; and was equipped with real-time contrast matching imaging technology (CnTI). SonoVue contrast agent (Bracco, Italy) was used.

\section{Image inspection steps}

Patients lay on a pillow in the supine position and the anterior neck area was fully exposed. First, conventional gray-scale ultrasound scans were performed, and the images were saved to record the size, location, internal echo, boundary, shape, and presence or absence of calcification of the thyroid nodules. Doppler ultrasound was used to detect the results of internal blood flow signal of the nodule, and then a preliminary assessment of the nodule.

\section{UE}

Firstly, two-dimensional ultrasound routine observation was conducted, and then the elastic mode was enabled on the longitudinal section. Two-dimensional ultrasound and elastic images were displayed on the left and right sides of the screen at the same time. When sampling, the area of interest included the lesion and the surrounding relatively normal thyroid tissue. The probe was held perpendicular to the lesion and vibrated slightly. The pressure and release action lasted for approximately 5 seconds until a more stable image was obtained.

According to the real-time flexible scoring standard $(15,16): 1$ point, the focus area is completely deformed after being compressed, and was entirely or mostly green; 2 points: most of the focus area is deformed after being compressed, and was mostly green and a small part of the center is blue; 3 points: after the focus area is compressed, a small part is deformed, and most of the center is blue and a small part of the periphery is green; 4 points, the lesion area is not deformed after compression, and the whole is uniform blue, or with a small amount of green; 5 points, there is no deformation in the focus area and surrounding areas after compression, and the focus and surrounding tissues are blue. A score of 1-3 was regarded as benign, and a score of $4-5$ was regarded as malignant.

\section{CEUS}

After the conventional two-dimensional observation, the contrast mode was activated, and the two-dimensional ultrasound and CEUS images were displayed on the left and right sides of the screen at the same time, so that the lesion could be compared with the surrounding normal thyroid tissue. The patient was instructed to breathe calmly and avoid swallowing. $2.4 \mathrm{~mL}$ of contrast agent was injected through the elbow vein, followed by $5.0 \mathrm{~mL}$ of $0.9 \%$ sodium chloride solution, which was observed in real time for 1-2 minutes. The dynamic recording of the contrast process was stored. The contrast enhancement mode of the lesion was recorded, uneven low enhancement or low concentric enhancement were diagnosed as malignant nodules, and high enhancement, equal enhancement, and no enhancement were treated as benign nodules.

\section{Image analysis}

Two physicians with more than 10 years of experience in ultrasound diagnosis independently analyzed the twodimensional sonographic features, CEUS, and elastic images of all nodules according to the above standards, and performed TI-RADS classification to determine benign and malignant. Another senior physician made the final interpretation.

\section{Foint scoring strategy combining CEUS and UE}

The elasticity score uses a modified 5 -point scoring standard: 1 point, the entire or most of the lesion is displayed in green; 2 points, the center of the lesion is displayed as blue, and the periphery is green; 3 points, the area of the lesion is displayed as green and blue in similar proportions; 4 points, the entire lesion is blue or with a little green inside; 5 points, the lesion and surrounding tissues are all shown in blue, with or without green inside. The ultrasound contrast mode was subsequently initiated and the double-frame contrast mode was adopted. After establishing 
Table 1 Grading of the joint scoring strategy combining CEUS and UE

\begin{tabular}{lcc}
\hline Inspection method & Image performance & Score \\
\hline UE & 1 score & 1 \\
& 2 scores & 2 \\
3 scores & 3 \\
& 4 scores & 4 \\
& 5 scores & 5 \\
CEUS & No enhancement & 1 \\
& Low enhancement & 2 \\
& Equal enhancement & 3 \\
& High enhancement & 4 \\
\hline
\end{tabular}

CEUS, contrast-enhanced ultrasound; UE, ultrasound elastography.

the superficial venous passage of the elbow, $1.2 \mathrm{~mL}$ of microbubble suspension was drawn for rapid injection. Next, $5 \mathrm{~mL}$ of normal saline was injected to flush the tube, and the timer was started at the same time to dynamically record the contrast. During the process, the patient was instructed to maintain the same position and breathe calmly throughout the radiography. The enhancement time, enhancement degree, enhancement mode and clearance time of the nodule were observed.

The evaluation indicators of CEUS were as follows: (I) the degree of lesion enhancement was divided into low enhancement, equal enhancement, high enhancement, and no enhancement; (II) enhancement mode (uniformity) was divided into uniform enhancement and uneven enhancement; and (III) clearance time was divided into rapid regression and slow regression.

Scores were assigned to each nodule. The combined scoring standards of CEUS and UE and elastography are shown in Table 1, and the total score of each nodule was calculated. The highest score was 9 points, and the lowest score was 2 points.

\section{Statistical analysis}

SPSS 24.0 (SPSS Inc. Chicago, IL, USA) was used for statistical analysis of the data. Count data were represented by examples (percent) [n (\%)]. Pearson's $\chi^{2}$ test was used for the theoretical number $T \geq 5$ and the total sample size $\mathrm{n} \geq 40$; continuous correction of $\chi^{2}$ test was used for the theoretical number $\mathrm{T}<5$ but $\mathrm{T} \geq 1$, and $\mathrm{n} \geq 40$; and Fisher's
Table 2 US-FNA cytology results

\begin{tabular}{lcc}
\hline Diagnostic result & Cases (n) & Percentage (\%) \\
\hline Cytologically diagnostic & 11 & 5.09 \\
Benign & 12 & 5.56 \\
Dysplasia & 22 & 10.19 \\
Follicular tumor & 30 & 13.89 \\
Suspiciously malignant & 101 & 46.76 \\
Malignant & 40 & 18.52 \\
Non-cytologically diagnostic & 216 & 100 \\
Total & & \\
\hline
\end{tabular}

US-FNA, ultrasound fine needle aspiration.

exact test was used for the theoretical number $\mathrm{T}<1$ or $\mathrm{n}<40$. Measurement data conforming to normal distribution were expressed as mean \pm standard deviation $(\bar{x} \pm s)$, which indicated that comparison between groups was performed with an independent sample $t$-test, and comparison within a group was performed with a paired sample $t$-test. Repeated measurement analysis of variance (ANOVA) was used to compare of multiple sets of measurement data. Both levels of $\alpha=0.05$ were used for the test level. For meaningful univariate parameters, multi-parameter logistic regression was used to analyze the independent risk coefficients. Receiver operating characteristic (ROC) curves and Kappa consistency checks were used to analyze the value of diagnosis. $\mathrm{P}<0.05$ was considered to be statistically significant.

\section{Results}

\section{US-FNA cytology results}

A total of 216 TI-RADS type 4 nodules were detected in 200 patients. Among them, 40 nodules were included in the non-cytologically diagnostic group, and 176 nodules were included in the cytologically diagnostic group. The cytological classifications are shown in Table 2.

\section{Independent risk factors of non-cytologically diagnostic thyroid nodules}

ANOVA analysis showed that compared with the cytologically diagnostic group, patients in the noncytologically diagnostic group had higher nodules echo, 
smaller aspect ratio, nodules calcifications were mostly peripheral, nodules were mostly irregular, and the depth of the lesion was closer to the middle and lower layers $(\mathrm{P}<0.05)$ (Table 3). The multi-parameter logistic regression showed that aspect ratio $<1$ [odds ratio $(\mathrm{OR})$ value $=2.90,95 \%$ confidence interval (CI): 1.92-4.33, $\mathrm{P}<0.001$ ], irregular edge $(\mathrm{OR}$ value $=1.52,95 \%$ CI: $0.97-2.09, \mathrm{P}=0.031)$, scattered coarse calcification $(\mathrm{OR}$ value $=2.01,95 \%$ CI: $1.23-5.44$, $\mathrm{P}=0.012)$, middle layer $(\mathrm{OR}$ value $=3.17,95 \%$ CI: $1.45-4.79$, $\mathrm{P}=0.011)$, and lower layer $(\mathrm{OR}$ value $=3.25$, 95\% CI: 1.87 $5.60, \mathrm{P}=0.005)$ were independent influencing factors leading to undiagnosed puncture cytology (Table 4).

\section{The efficacy of the joint scoring strategy in diagnosing benign and malignant nodules}

Among the 216 nodules in 200 patients, 168 were pathologically diagnosed as malignant nodules (malignant group), and 48 nodules were diagnosed as benign nodules (benign group). According to the joint scoring strategy, the number of nodules in different segments is shown in Table 5 . In benign nodules, the distribution of comprehensive scores changes significantly at 5-6 scores, while in malignant nodules, the distribution of comprehensive scores changes significantly at 6-7 scores. Therefore, we used 5, 6, and 7 scores as the critical points for differential diagnosis, and the results of ROC curve analysis showed that the diagnostic value was the highest when ' 6 scores' was used as the critical point for diagnosis [area under curve (AUC) $=0.893, \mathrm{P}<0.05]$ (Figure 1$)$.

\section{Discussion}

At present, conventional ultrasound is the first-line option to identify benign and malignant thyroid nodules. However, because the sonographic features of thyroid nodules are more complicated, and the signs between benign and malignant nodules often overlap, certain difficulties to the further improvement of the differential diagnosis of benign and malignant thyroid nodules arise. The emergence of TI-RADS classification standard provides a feasible and effective criterion for the standardization of diagnosis; however, it is not a diagnostic standard to accurately define benign and malignant nodules, especially for TI-RADS 4-type nodules (17). UG-FNA is a convenient, safe, and effective method to identify the nature of thyroid nodules recommended by the thyroid diagnosis and treatment guidelines (18), and to analyze the factors that make
UG-FNA undiagnosable is of great significance. It can reduce unnecessary puncture, reasonably allocate medical resources, and reduce the suffering of patients. Previous studies have reported that the undiagnosable rate of thyroid nodules under ultrasound guidance is $3-21 \%$ (19), and the undiagnosable rate of thyroid nodules in this study was $18.5 \%$, which is consistent with the previous reports.

In this study, we identified independent risk factors that cannot be diagnosed by UG-FNA for TI-RADS type 4 nodules, including aspect ratio $<1$, irregular edge, scattered coarse calcification, middle layer, and lower layer. The influencing factors of TI-RADS 4-type nodules that cannot be diagnosed by UG-FNA are controversial. Previous studies have disputed about the effect of echo on the rate of undiagnosed puncture; some studies found that the effect of echo on the rate of undiagnosed puncture is not statistically significant (20). There are also studies reporting that hypoechoic is an independent factor (21) that predicts that the pathology of puncture cannot be diagnosed. The present study proposed that echo is a related influencing factor that causes cytology to be undiagnosed and an independent influencing factor. Echo is formed by tissues receiving sound waves and constantly reflecting them. The internal composition and cell structure of different nodules are different, as is the formation of the reflective interface, which exhibits different echoes. Fibrosis, inflammatory cell infiltration, etc. are not conducive to the acquisition of thyroid follicular epithelial cells via needle tube, so we believe that echo has an impact on the failure rate of puncture. Choi et al. (22) concluded that the aspect ratio has no effect on the failure rate of puncture, but our study reached the opposite conclusion. This finding requires further confirmation by increasing the amount of data and multi-center studies. Few studies have focused on the relationship between the depth of nodules and the undiagnosable rate of puncture cytology. Xia et al. (23) reported that the depth of nodules $>15 \mathrm{~mm}$ is an independent factor leading to undiagnosed cytology. In the present study, we found that the location of the nodules in the middle and posterior layers is an independent influencing factor that makes the cytological results undiagnosable. The closer the nodules are located to the dorsal side, the higher the rate of undiagnosed puncture. This may be due to the deeper the position of the nodule, the longer puncture approach, and other influencing factors, such as the resistance of muscles, thyroid, and other tissues, as well as the side pressure caused by the slope of the puncture needle. Secondly, the length of the needle 
Table 3 ANOVA between the cytologically diagnostic and non-cytologically diagnostic groups

\begin{tabular}{|c|c|c|c|c|}
\hline Factors & Cases (n) & Cytologically diagnostic group $(n=176)$ & Non-cytologically diagnostic group $(n=40)$ & $P$ value \\
\hline$\leq 5 \mathrm{~mm}$ & 48 & $38(79.17)$ & $10(20.83)$ & \\
\hline $5-10 \mathrm{~mm}$ & 122 & $98(80.33)$ & $24(19.67)$ & \\
\hline$\geq 10 \mathrm{~mm}$ & 46 & $40(86.96)$ & $6(13.04)$ & \\
\hline None & 6 & $5(83.33)$ & $1(16.67)$ & \\
\hline Low & 200 & $165(82.50)$ & $35(17.50)$ & \\
\hline Equal & 7 & $4(57.14)$ & $3(42.86)$ & \\
\hline High & 3 & $2(66.67)$ & $1(33.33)$ & \\
\hline$<1$ & 98 & $73(74.49)$ & $25(25.51)$ & \\
\hline Calcification & & & & 0.001 \\
\hline Peripheral & 9 & $6(66.67)$ & $3(33.33)$ & \\
\hline Scattered coarse & 32 & $23(71.88)$ & $9(28.12)$ & \\
\hline Scattered small & 77 & 67 (87.01) & 10 (12.99) & \\
\hline None & 98 & 80 (81.63) & 18 (18.37) & \\
\hline Edge characteristics & & & & 0.001 \\
\hline Regular & 54 & $39(72.22)$ & 15 (27.78) & \\
\hline Depth & & & & 0.004 \\
\hline Upper & 57 & $50(87.72)$ & $7(12.28)$ & \\
\hline Middle & 108 & 86 (79.63) & $22(20.37)$ & \\
\hline Lower & 36 & 26 (72.22) & $10(27.78)$ & \\
\hline Isthmus & 15 & 14 (93.33) & $1(6.67)$ & \\
\hline Adjacent to & & & & 0.152 \\
\hline Inside & 22 & $19(86.36)$ & $3(13.64)$ & \\
\hline Midside & 153 & $122(79.74)$ & $31(20.26)$ & \\
\hline Outside & 26 & $21(80.77)$ & $5(19.23)$ & \\
\hline Isthmus & 15 & 14 (93.33) & $1(6.67)$ & \\
\hline
\end{tabular}


Table 4 Multi-parameter logistic regression of the non-cytologically diagnostic group

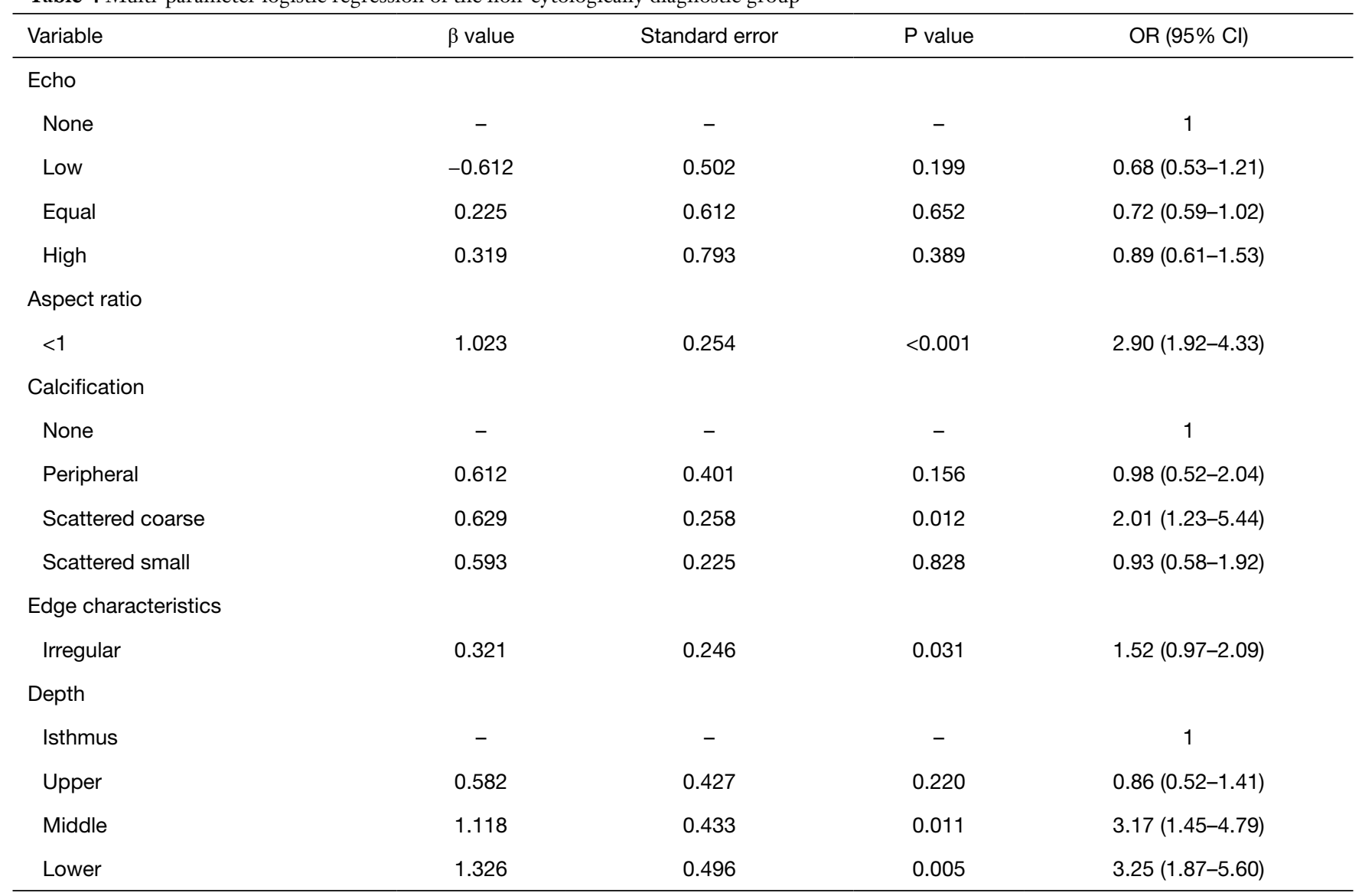

Table 5 Scores distribution of nodules according to the joint scoring strategy combining CEUS and UE

\begin{tabular}{lccc}
\hline Pathological diagnosis & $\begin{array}{c}\text { Benign } \\
\text { group }\end{array}$ & $\begin{array}{c}\text { Malignant } \\
\text { group }\end{array}$ & Total \\
\hline Scores distribution & 4 & 0 & 4 \\
2 & 8 & 1 & 9 \\
3 & 12 & 5 & 17 \\
4 & 18 & 14 & 32 \\
5 & 3 & 25 & 28 \\
6 & 2 & 42 & 44 \\
7 & 1 & 55 & 56 \\
8 & 0 & 26 & 26 \\
9 & 48 & 168 & 216 \\
Total & & &
\end{tabular}

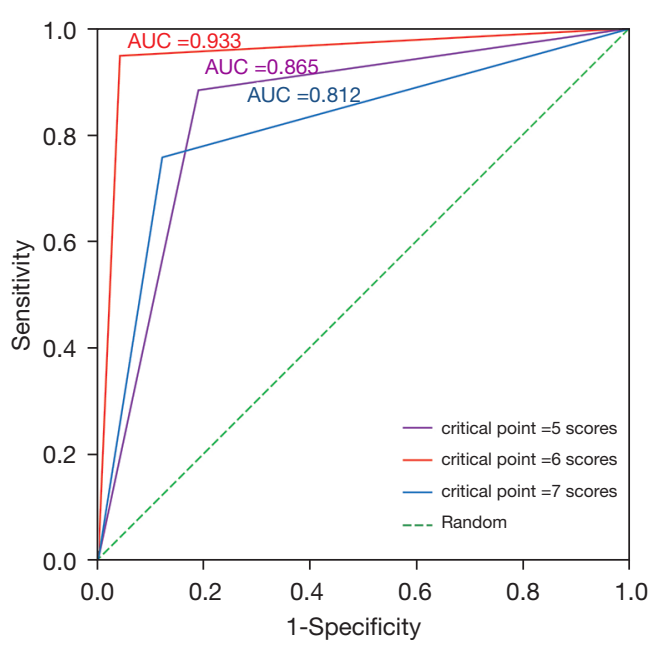

Figure 1 Diagnostic effect of different joint scoring scores as critical points for differential diagnosis between benign and malignant nodules. 
tube limits the lifting range of the puncture needle, which may result in the reduction of effective tissue in the needle tube. In addition, our research also confirmed that irregular edge and scattered coarse calcification are independent risk factors that cause cytology to be undiagnosable. However, more research is required to confirm these findings.

Although the application value of UE in the differential diagnosis of benign and malignant thyroid nodules has been recognized, due to the diversity and complexity of the nodules themselves, elastography also has certain limitations, resulting in some false negative and false positive cases. Huang et al. (24) found that UE to diagnose TIRADS 4 nodules has an accuracy rate of up to $90 \%$, while Kovatcheva et al. (25) reported an accuracy rate of only $76 \%$. CEUS has been a hot spot in the field of ultrasound research in recent years. Its application in abdominal solid organs such as the liver is relatively mature; however, its application in superficial organs is still in the exploratory stage, especially for the differential diagnosis of benign and malignant thyroid nodules. There is considerable controversy, and the classification of imaging modes has not yet formed a unified standard. Zhao et al. (26) reported that the accuracy of CEUS in diagnosing TI-RADS 4 nodules reached $90 \%$, and the study of Zhao et al. (27) observed that the AUC of CEUS in diagnosing TI-RADS 4 nodules was 0.8. It is worth noting that the gold standard for diagnosis of patients in this study was UG-FNA. However, the aforementioned previous studies did not pay attention to whether TI-RADS 4 nodules are undiagnosable by UGFNA. In this study, we integrated UE and CEUS, and a novel scoring strategy was constructed by means of joint scoring. We used a combined score of 6 as the critical point of diagnosis, and finally obtained an AUC of 0.933 , which was higher than the previous report. The research of Lin et al. (28) confirmed the value of UE combined with CEUS in diagnosing the TI-RADS4 nodules that could not be diagnosed by UG-FNAC, which combined the diagnosis results of CEUS and UE in parallel to improving the sensitivity of TI-RADS 4 types of nodules. The difference is that our study uses the CEUS and UE parameters of the nodules as scoring items to develop a joint scoring strategy. The scoring strategy can more fully combine the two imaging inspection methods, and the experimental results prove that our diagnosis method had obtained a higher AUC value than Lin et al. (28). This indicates the combined scoring strategy of CEUS and UE is an effective choice.

In summary, an aspect ratio $<1$, irregular edge, scattered coarse calcification, middle layer, and lower layer are independent risk factors that UG-FNA cannot diagnose. For patients who are unable to perform cytological diagnosis, the combined scoring strategy of CEUS and UE is an effective choice. The limitations of this study were as follows: (I) the amount of data we studied is limited, which may lead to a certain bias in our conclusions; and (II) due to the insufficient amount of data, our study did not diagnose benign and malignant TI-RADS4 nodules that cannot be diagnosed with UG-FNA using the combined scoring strategy.

\section{Acknowledgments}

Funding: None.

\section{Footnote}

Reporting Checklist: The authors have completed the STARD reporting checklist. Available at https://dx.doi. org/10.21037/gs-21-512

Data Sharing Statement: Available at https://dx.doi. org/10.21037/gs-21-512

Conflicts of Interest: All authors have completed the ICMJE uniform disclosure form (available at https://dx.doi. org/10.21037/gs-21-512). The authors have no conflicts of interest to declare.

Ethical Statement: The authors are accountable for all aspects of the work in ensuring that questions related to the accuracy or integrity of any part of the work are appropriately investigated and resolved. All procedures performed in this study involving human participants were in accordance with the Declaration of Helsinki (as revised in 2013). This study was approved by the Medical Ethics Committee of Xiamen Hospital of Traditional Chinese Medicine (No. EC20200808A) and informed consent was taken from all the patients.

Open Access Statement: This is an Open Access article distributed in accordance with the Creative Commons Attribution-NonCommercial-NoDerivs 4.0 International License (CC BY-NC-ND 4.0), which permits the noncommercial replication and distribution of the article with the strict proviso that no changes or edits are made and the original work is properly cited (including links to both the formal publication through the relevant DOI and the license). 
See: https://creativecommons.org/licenses/by-nc-nd/4.0/.

\section{References}

1. Seib CD, Sosa JA. Evolving Understanding of the Epidemiology of Thyroid Cancer. Endocrinol Metab Clin North Am 2019;48:23-35.

2. Massimino M, Evans DB, Podda M, et al. Thyroid cancer in adolescents and young adults. Pediatr Blood Cancer 2018;65:e27025.

3. Wong R, Farrell SG, Grossmann M. Thyroid nodules: diagnosis and management. Med J Aust 2018;209:92-8.

4. Zhang L, Gu J, Zhao Y, et al. The role of multimodal ultrasonic flow imaging in Thyroid Imaging Reporting and Data System (TI-RADS) 4 nodules. Gland Surg 2020;9:1469-77.

5. Gao L, Xi X, Jiang Y, et al. Comparison among TIRADS (ACR TI-RADS and KWAK- TI-RADS) and 2015 ATA Guidelines in the diagnostic efficiency of thyroid nodules. Endocrine 2019;64:90-6.

6. Wang J, Liu J, Liu Z. Impact of ultrasound-guided fine needle aspiration cytology for diagnosis of thyroid nodules. Medicine (Baltimore) 2019;98:e17192.

7. Wang J, Bai XG, Liu Z. Impact of ultrasound angiography combined with fine needle aspiration for the diagnosis of thyroid nodules. Medicine (Baltimore) 2019;98:e17286.

8. Suh CH, Baek JH, Lee JH, et al. The role of core-needle biopsy in the diagnosis of thyroid malignancy in 4580 patients with 4746 thyroid nodules: a systematic review and meta-analysis. Endocrine 2016;54:315-28.

9. Shi YX, Chen L, Liu YC, et al. Differences among the Thyroid Imaging Reporting and Data System proposed by Korean, the American College of Radiology and the European Thyroid Association in the diagnostic performance of thyroid nodules. Transl Cancer Res 2020;9:4958-67.

10. Tessler FN, Middleton WD, Grant EG, et al. ACR Thyroid Imaging, Reporting and Data System (TI-RADS): White Paper of the ACR TI-RADS Committee. J Am Coll Radiol 2017;14:587-95.

11. Fradin JM. ACR TI-RADS: An advance in the management of thyroid nodules or Pandora's box of surveillance? J Clin Ultrasound 2020;48:3-6.

12. Tessler FN, Middleton WD, Grant EG. Thyroid Imaging Reporting and Data System (TI-RADS): A User's Guide. Radiology 2018;287:29-36.

13. Yang JR, Song Y, Xue SS, et al. Suggested amendment of TI-RADS classification of thyroid nodules by shear wave elastography. Acta Radiol 2020;61:1026-33.

14. Tan $\mathrm{H}, \mathrm{Li} Z, \mathrm{Li}$, et al. Thyroid imaging reporting and data system combined with Bethesda classification in qualitative thyroid nodule diagnosis. Medicine (Baltimore) 2019;98:e18320.

15. Fang H, Gong L, Xu Y, et al. Reliable Thyroid Carcinoma Detection with Real-Time Intelligent Analysis of Ultrasound Images. Ultrasound Med Biol 2021;47:590-602.

16. Trimboli P, Paone G, Zatelli MC, et al. Real-time elastography in autonomously functioning thyroid nodules: relationship with TSH levels, scintigraphy, and ultrasound patterns. Endocrine 2017;58:488-94.

17. Pei S, Cong S, Zhang B, et al. Diagnostic value of multimodal ultrasound imaging in differentiating benign and malignant TI-RADS category 4 nodules. Int J Clin Oncol 2019;24:632-9.

18. Nabhan F, Ringel MD. Thyroid nodules and cancer management guidelines: comparisons and controversies. Endocr Relat Cancer 2017;24:R13-26.

19. Brister KJ, Singh RS, Wang HH. Reporting thyroid FNA before and after implementation of the Bethesda system-one institution's experience. Diagn Cytopathol 2015;43:28-31.

20. Degirmenci B, Haktanir A, Albayrak R, et al. Sonographically guided fine-needle biopsy of thyroid nodules: the effects of nodule characteristics, sampling technique, and needle size on the adequacy of cytological material. Clin Radiol 2007;62:798-803.

21. Wu H, Zhang B, Zang Y, et al. Ultrasound-guided fineneedle aspiration for solid thyroid nodules larger than $10 \mathrm{~mm}$ : correlation between sonographic characteristics at the needle tip and nondiagnostic results. Endocrine 2014;46:272-8.

22. Choi SH, Han KH, Yoon JH, et al. Factors affecting inadequate sampling of ultrasound-guided fine-needle aspiration biopsy of thyroid nodules. Clin Endocrinol (Oxf) 2011;74:776-82.

23. Xia JJ, Li MS, Zheng L, et al. Nondiagnostic cytological results on ultrasound-guided fine needle aspiration: Does the thyroid nodule depth matter? Clin Hemorheol Microcirc 2017;67:115-24.

24. Huang S, Meng N, Pan M, et al. Diagnostic performances of the KWAK-TIRADS classification, elasticity score, and Bethesda System for Reporting Thyroid Cytopathology of TI-RADS category 4 thyroid nodules. Int J Clin Exp Pathol 2020;13:1159-68.

25. Kovatcheva RD, Shinkov AD, Dimitrova ID, et al. 
Evaluation of the Diagnostic Performance of EU-TIRADS in Discriminating Benign from Malignant Thyroid Nodules: A Prospective Study in One Referral Center. Eur Thyroid J 2021;9:304-12.

26. Zhao Y, Zhou P, Peng H, et al. Application of superb microvascular imaging and contrast enhanced ultrasound in the differential diagnosis of thyroid nodules. Zhong Nan Da Xue Xue Bao Yi Xue Ban 2019;44:649-56.

27. Zhao H, Liu X, Lei B, et al. Diagnostic performance of thyroid imaging reporting and data system (TI-RADS)

Cite this article as: Jiang C, Chang J, Chen X, Zhang H, Xu M. Independent risk factors and feasibility of ultrasound diagnosis of ultrasound-guided non-cytologically diagnostic thyroid nodules. Gland Surg 2021;10(9):2724-2733. doi: 10.21037/gs21-512 alone and in combination with contrast-enhanced ultrasonography for the characterization of thyroid nodules. Clin Hemorheol Microcirc 2019;72:95-106.

28. Lin ZM, Wen Q, Yan CX, et al. Combination of contrastenhanced ultrasound and strain elastography to assess cytologically non-diagnostic thyroid nodules. Oncol Lett 2019;18:6845-51.

(English Language Editor: A. Kassem) 